\title{
Florística e fitossociologia do componente arbóreo de uma floresta ribeirinha, arroio Passo das Tropas, Santa Maria, RS, Brasil
}

\author{
Jean Carlos Budke ${ }^{1,2}$, Eduardo Luis Hettwer Giehl' ${ }^{1}$, Eduardo Anversa Athayde ${ }^{1}$, \\ Sônia Maria Eisinger ${ }^{1}$ e Renato Aquino Záchia ${ }^{1,2}$
}

Recebido em 22/08/2003. Aceito em 04/02/2004

\begin{abstract}
RESUMO - (Florística e fitossociologia do componente arbóreo de uma floresta ribeirinha, arroio Passo das Tropas, Santa Maria, RS, Brasil). Florestas ribeirinhas são caracterizadas por alta heterogeneidade ambiental, onde diferentes fatores físicos e bióticos regulam o mosaico vegetacional. Embora sejam áreas prioritárias à conservação, devido a sua fragilidade e importância, poucos estudos vêm sendo desenvolvidos nestes ambientes, em especial no Rio Grande do Sul. O objetivo do presente estudo é caracterizar a composição florística e a estrutura fitossociológica de um trecho de floresta ribeirinha em Santa Maria, RS. Para o levantamento, foram demarcadas 100 parcelas de $10 \times 10 \mathrm{~m}$ cada, dispostas paralelamente ao curso do rio, onde todos os indivíduos arbóreos PAP $\geq 15 \mathrm{~cm}$ foram amostrados. Foram amostrados 2.195 indivíduos vivos e mais 137 mortos ainda em pé. Os indivíduos vivos pertencem a 57 espécies de 47 gêneros distribuídos em 26 famílias. As espécies com maiores valores de importância foram Gymnanthes concolor Spreng, Sebastiania commersoniana (Baill.) Smith \& Downs, Eugenia uniflora L., Plinia rivularis (Camb.) Rotman e Sebastiania brasiliensis Spreng. O índice de diversidade (H') foi de 2,73 nats/indivíduos e a equabilidade (J') 0,69 nats/indivíduos, valores considerados intermediários em comparação a outros levantamentos. A maioria das espécies amostradas (57\%) provém do oeste do Estado, características das bacias do Paraná-Uruguai, enquanto que $41 \%$ são de ampla distribuição e apenas uma espécie provém do corredor atlântico.
\end{abstract}

Palavras-chave: estrutura da comunidade, florestas ribeirinhas, fitogeografia, rota de migração de plantas

\begin{abstract}
Floristic composition and structure of arboreal species in a riverine forest, Passo das Tropas River, Santa Maria, RS, Brazil). Riverine forests have great environmental diversity, where many factors, physical and biotical, play a role on the vegetation mosaic. Although riverine forests are hot spots for conservation, because of their fragility and ecological importance, few studies has been made in these areas, especially in Rio Grande do Sul State. The aim of this work is to determine the floristic composition and the vegetation structure of arboreal species in a riverine forest in Santa Maria municipality. All individuals with $\mathrm{PBH} \geq 15 \mathrm{~cm}$ were sampled within 100 plots, each one $100 \mathrm{~m}^{2}$. A total of 2,195 living and 137 standing dead individuals were sampled, belonging to 57 species and 26 botanicals families. The species with the largest importance values are Gymnanthes concolor Spreng, Sebastiania commersoniana (Baill.) Smith \& Downs, Eugenia uniflora L., Plinia rivularis (Camb.) Rotman and Sebastiania brasiliensis Spreng. The diversity index $\left(H^{\prime}\right)$ was 2.73 nats/individuals and the equability (J') 0.69 nats/individuals, which are considered intermediates values when compared with others works. More than half of the species (57\%) belong to the eastern migratory group, characteristic of the Paraná-Uruguai basin, while $41 \%$ of the species have a large distribution in the State and only one specie belong to the Atlantic corridor.
\end{abstract}

Key words: community structure, riverine forests, phytogeography, plant migration routes

\section{Introdução}

Matas ribeirinhas são formações florestais encontradas ao longo de cursos d'água, sendo caracterizadas por alta heterogeneidade ambiental. Diversos fatores físicos e bióticos atuam sobre elas, modificando as características do ambiente e regulando assim, o mosaico vegetacional (Rodrigues $\&$ Shepherd 2000).

Em função da forte pressão humana sobre estes ambientes, vários trabalhos vêm sendo desenvolvidos em diferentes regiões do país, especialmente nas regiões Sudeste (Rodrigues \& Nave 2000; Bertani et al. 2001; Toniato et al. 1998; Rodrigues 2000) e Centro-Oeste (van der Berg \& Oliveira Filho 2000; Oliveira Filho et al. 1990; Ribeiro \& Walter 1998) com o intuito de obterse informações sobre os padrões fitogeográficos existentes e/ou dados ecológicos que possam auxiliar na recuperação de paisagens degradadas (Leite 2001; Botrel et al. 2002; Moreno \& Schiavini 2001).

Na região da Depressão Central, assim como em todo o Rio Grande do Sul, ainda são escassos os estudos

\footnotetext{
Departamento Biologia, Universidade Federal de Santa Maria, Faixa de Camobi, Km 09, CEP 97105-200, Santa Maria, RS, Brasil

2 Autor para correspondência: jeancbpb@zipmail.com
} 
realizados em florestas ribeirinhas (Daniel 1991). Esta escassez de estudos não tem permitido uma compreensão sobre a dinâmica destas florestas, mas sim de aspectos florístico-geográficos ou estruturais.

Segundo Jarenkow \& Waechter (2001) muitos trabalhos têm sido realizados objetivando caracterização fitogeográfica das distintas formações florestais do Estado. Rambo (1951) foi o pioneiro a realizar este tipo de abordagem, caracterizando duas principais rotas de migração de espécies vegetais no Rio Grande do Sul, denominadas "Porta de Torres", abrangendo as espécies da porção leste, ou seja, o elemento atlântico, proveniente de latitudes menores e, "Alto Uruguai", com as espécies que compõem as florestas dos rios Paraná e Uruguai. Entre estas duas formações, existe o Planalto, de altitude mais elevada e conseqüentemente, temperaturas médias inferiores, o que dificulta a sobreposição destes dois contingentes (Jarenkow \& Waechter 2001).

Rotas de migração para formações ribeirinhas foram pouco questionadas, pois formações vegetacionais associadas a cursos d'água são tratadas na literatura com nomenclatura tão variada quanto confusa (Van der Berg \& Oliveira Filho 2000), resultado de grandes variações florísticas e estruturais encontradas, não havendo, assim, uma terminologia adequada e prevalecendo muitas vezes, os nomes populares. Rodrigues (2000) realizou uma revisão sobre esta terminologia, chegando a propor uma nova base nomenclatural a fim de melhor caracterizar as formações florestais ribeirinhas.

Os objetivos do presente estudo foram conhecer a composição florística, a estrutura fitossociológica e as possíveis rotas de migração das plantas de um trecho de floresta ribeirinha em Santa Maria, Rio Grande do Sul, contribuindo para o conhecimento florísticoestrutural e fitogeográfico das formações florestais da região.

\section{Material e métodos}

Caracterização da área de estudo - Santa Maria está localizada na região da Depressão Central, no Estado do Rio Grande do Sul. A área de estudo é um trecho de floresta ribeirinha, pertencente à Universidade Federal de Santa Maria - UFSM, localizada em torno das coordenadas $53^{\circ} 45^{\prime} \mathrm{W}$ e $29^{\circ} 45^{\prime} \mathrm{S}$, na divisa do campus com uma vila urbana. Tabarelli (1992) considerou estas formações pela descrição de Floresta Decídua Baixo-Montana, como partes componentes da unidade fitofisionômica da Floresta Estacional
Decidual, seguindo a classificação proposta pelo Projeto RADAMBRASIL (Veloso et al. 1991).

O rio Passo das Tropas é um pequeno afluente da microbacia do rio Vacacaí-mirim, possuindo poucos remanescentes vegetais ao longo do seu curso, devido à intensa exploração agrícola. A área de estudo está inalterada há pelo menos 50 anos, possuindo em torno de 20 ha de floresta contínua. A declividade do terreno é baixa, sendo comum o alagamento parcial da área quando ocorre extravasamento do leito do rio. Esta característica faz com que estes córregos sejam popularmente conhecidos por "arroios".

O clima da região é do tipo Cfa (subtropical úmido), de acordo com a classificação climática de Köppen (Moreno 1961; Maluf 2000). Dados provenientes da Estação de Meteorologia de Santa Maria, localizada no campus da UFSM, a 138m de altitude, no período de 1961-1990, revelam temperatura média anual de $19,6^{\circ} \mathrm{C}$, com médias mensais variando de $24,8^{\circ} \mathrm{C}$ em janeiro a $14,3^{\circ} \mathrm{C}$ em junho; precipitação media anual de $1.686 \mathrm{~mm}$, com chuvas bem distribuídas durante o ano.

Os solos pertencem à unidade de Mapeamento Santa Maria, sendo classificados como Brunizem Hidromórfico (textura média, terreno suavemente ondulado, substrato areia-siltito). Esta Unidade de Mapeamento é constituída por solos medianamente profundos, textura superficial argilosa e drenagem imperfeita (Lemos et al. 1973).

Levantamento florístico e fitossociológico - A demarcação da área e o posterior levantamento transcorreram entre agosto/2001 e agosto/2002. Foram demarcadas 100 parcelas de $10 \times 10 \mathrm{~m}$ cada, divididos em transectos de $10 \times 100 \mathrm{~m}$. Os transectos foram dispostos paralelamente ao curso do arroio, com uma distância de dez metros entre cada transecto, não havendo mudança topográfica perceptível. Este critério provém da dificuldade de se encontrar uma área com largura de mais de $100 \mathrm{~m}$ desde a borda do arroio até a margem da floresta.

Em cada parcela, foram amostrados todos os indivíduos arbóreos, com perímetro à altura do peito (PAP) igual ou superior a $15 \mathrm{~cm}$. Árvores mortas ainda em pé, presentes nas parcelas e com critério mínimo de inclusão, foram amostradas e colocadas em uma classe única, não participando dos cálculos vegetacionais. O material fértil coletado foi etiquetado, identificado e herborizado no Laboratório de Sistemática Vegetal (LABSIS), sendo depositado posteriormente no herbário SMDB, UFSM. As 
espécies foram agrupadas nas famílias reconhecidas pelo APG, Angiosperm Phylogeny Group (1998).

Análise dos dados - Os dados foram analisados com o auxílio do programa FITOPAC (Shepherd 1995), obtendo-se os parâmetros fitossociológicos usuais calculados em Fitossociologia (Densidade, Freqüência e Dominância - absolutas e relativas, Valor de Importância e Valor de Cobertura). A diversidade específica foi determinada utilizando o índice de Shannon (H') e o índice de equabilidade de Pielou (J') (Brower \& Zar 1984).

Para se avaliar o contingente de migração, as espécies foram separadas de acordo com os corredores seguidos para chegarem no Rio Grande do Sul, seguindo metodologia de Jarenkow \& Waechter (2001). Assim, com base na bibliografia especializada (Reitz et al. 1983; Klein 1972; Rambo 1961; Tabarelli 1992; Jarenkow 1994; Jarenkow \& Waechter 2001) as espécies foram caracterizadas como pertencentes ao corredor atlântico (leste) ou ao longo das bacias dos rios Paraná e Uruguai (oeste), excluindo-se a região dos pinhais. Espécies de ampla distribuição foram separadas numa classe distinta.

\section{Resultados e discussão}

Foram amostrados 2.195 indivíduos vivos e 137 indivíduos mortos ainda em pé. Os indivíduos vivos distribuiram-se em 57 espécies de 47 gêneros, pertencentes a 26 famílias botânicas. Uma espécie permaneceu não identificada devido à falta de material fértil. As espécies, famílias, números de coleta, respectivos corredores migratórios e parâmetros fitossociológicos estão listados na Tab. 1.

A família com maior número de espécies amostradas foi Myrtaceae (17), sendo uma das famílias com maior riqueza de espécies arbóreas amostradas em levantamentos no Estado (Jarenkow 1994; Jarenkow \& Baptista 1987). Rambo (1949; 1951) apontou esta família como sendo de grande importância na riqueza das matas com araucária. $\mathrm{O}$ mesmo tem sido diagnosticado para as outras formações florestais do Rio Grande do Sul (Jarenkow \& Waechter 2001; Longhi et al. 1999). A segunda família com maior riqueza foi Fabaceae (6), seguida por Euphorbiaceae, Flacourtiaceae e Rubiaceae com três espécies cada. Eugenia (6), Casearia, Sebastiania e Myrsine (2) foram os gêneros com maiores riquezas em espécies. Os demais 43 gêneros apresentaram uma única espécie cada.
A densidade total, 2.195 ind./ha, foi considerada elevada, em comparação com outros levantamentos da região (Jarenkow \& Waechter 2001; Longhi et al. 1999; Daniel 1991). A maioria dos indivíduos (1.762 ind., equivalendo a $80 \%$ ) pertencia a apenas três famílias, principalmente Euphorbiaceae (991 ind.), seguida de Myrtaceae (698 ind.) e Rubiaceae (73 ind.).

As espécies com maiores valores de densidade relativa (DR) foram Gymnanthes concolor $(32,57)$, Eugenia uniflora $(9,84)$, Plinia rivularis $(8,79)$, Sebastiania commersoniana $(7,97)$ e Sebastiania brasiliensis $(4,70)$, correspondendo a $63,78 \%$ do total de indivíduos amostrados. Estas posições alteram-se quando é analisado o valor de freqüência absoluta, encontrando-se em primeira posição Eugenia uniflora (82) seguida de Gymnanthes concolor (81), Sebastiania commersoniana (71), Plinia rivularis (64) e Sebastiania brasiliensis (53). A grande maioria das espécies ocorreu em baixas frequiências sendo que 42 espécies $(73,68 \%)$ estão presentes em menos de $20 \%$ das parcelas.

A relação entre número de indivíduos e área amostrada está representada na Fig. 1. A curva ajustada por regressão logarítmica apresenta tendência à estabilização, sendo que a partir da $50^{\circ}$ parcela, apenas 8 espécies foram acrescidas, ou seja, aumentando-se mais que $10 \%$ de área, não houve acréscimo correspondente $(5 \%)$ no número de espécies. Incremento acentuado de espécies ocorreu entre as parcelas 93 e 96, provavelmente em função da sua localização, mais próximas da borda. O número máximo de indivíduos amostrados em cada parcela foi de 50 e o mínimo, nove indivíduos.

Quanto à área basal, os maiores valores foram de Sebastiania commersoniana $(3,662)$, Plinia rivularis (3,046), Gymnanthes concolor (2,487), Eugenia uniflora $(2,307)$ e Sebastiania brasiliensis $(1,762)$. Gymanthes concolor apresentou elevada densidade, o que confere a esta espécie o maior valor de importância (VI). No entanto, é espécie típica do subbosque, com indivíduos de pequena área basal, colocando-a em terceira posição quando o parâmetro foi analisado isoladamente. Por outro lado, Sebastiania commersoniana que ficou na quarta posição em termos de densidade relativa, apresentou a maior área basal devido ao elevado diâmetro dos seus indivíduos, quando comparado às outras espécies.

Gymnanthes concolor $(50,61)$, seguida por Sebastiania commersoniana $(29,26)$, Eugenia uniflora (27,33), Plinia rivularis $(27,04)$ e Sebastiania brasiliensis $(16,90)$ apresentaram os maiores VI e 
Tabela 1. Famílias, espécies e respectivos números de coleta e parâmetros vegetacionais de uma área de floresta ribeirinha do arroio Passo das Tropas, Santa Maria, RS ordenadas por ordem de importância das famílias. DA = Densidade absoluta (ind. ha ${ }^{-1}$ ); DoA = Dominância absoluta $\left(\mathrm{m}^{2} . \mathrm{ha}^{-1}\right) ; \mathrm{FA}=$ Freqüência absoluta $(\%) ; \mathrm{VC}=$ Valor de cobertura $(\%) ; \mathrm{VI}=$ Valor de importância $(\%) ; \mathrm{NC}=$ número do coletor; $\mathrm{CG}=$ contingente geográfico da espécie, sendo que EAD = espécie de ampla distribuição; BPU = característica da Bacia Paraná-Uruguai; ATL $=$ corredor atlântico.

\begin{tabular}{|c|c|c|c|c|c|c|c|}
\hline Família/Espécie & $\mathrm{NC}$ & CG & $\mathrm{DA}$ & DoA & FA & $\mathrm{VC}$ & VI \\
\hline EUPHORBIACEAE & & & 991 & 7,911 & 98 & 37,18 & 32,18 \\
\hline Gymnanthes concolor Spreng. & JCB 303 & EAD & 715 & 2,487 & 81 & 20,88 & 16,80 \\
\hline Sebastiania commersoniana (Baill.) Smith \& Downs & JCB 304 & EAD & 101 & 3,662 & 71 & 10,75 & 9,75 \\
\hline Sebastiania brasiliensis Spreng. & $\mathrm{NC}$ & EAD & 175 & 1,762 & 53 & 5,55 & 5,63 \\
\hline MYRTACEAE & & & 698 & 9,344 & 97 & 33,14 & 34,41 \\
\hline Eugenia uniflora $\mathrm{L}$. & JCB 115 & EAD & 216 & 2,309 & 82 & 9,18 & 9,11 \\
\hline Plinia rivularis (Camb.) Rotman & JCB 118 & BPU & 193 & 3,046 & 64 & 10,02 & 9,01 \\
\hline Eugenia uruguayensis Camb. & JCB 125 & BPU & 61 & 0,746 & 35 & 2,77 & 3,12 \\
\hline Myrceugenia glaucescens (Camb.) Legr. \& Kaus. & JCB 127 & BPU & 69 & 0,748 & 31 & 2,95 & 3,09 \\
\hline Eugenia hyemalis Camb. & JCB 114 & ATL & 42 & 0,294 & 25 & 1,50 & 1,91 \\
\hline Calyptranthes conccina DC. & JCB 392 & BPU & 18 & 0,285 & 15 & 0,93 & 1,17 \\
\hline Gomidesia palustris (DC.) Kaus. & JCB 216 & $\mathrm{BPU}$ & 14 & 0,342 & 14 & 0,95 & 1,14 \\
\hline Eugenia involucrata DC. & JCB 153 & BPU & 13 & 0,364 & 13 & 0,97 & 1,12 \\
\hline Eugenia ramboi Legr. & JCB 135 & BPU & 15 & 0,244 & 15 & 0,79 & 1,07 \\
\hline Blepharocalyx salicifolius (Kunth) Berg. & $\mathrm{NC}$ & BPU & 9 & 0,202 & 9 & 0,58 & 0,71 \\
\hline Campomanesia xanthocarpa Berg. & JCB 179 & EAD & 12 & 0,257 & 9 & 0,75 & 0,82 \\
\hline Myrcianthes pungens (Berg.) Legr. & JCB 178 & BPU & 13 & 0,212 & 10 & 0,69 & 0,82 \\
\hline Myrcia bombycina (Berg.) Niedenzu & JCB 263 & BPU & 9 & 0,052 & 5 & 0,30 & 0,38 \\
\hline Eugenia rostrifolia Legr. & JCB 275 & $\mathrm{BPU}$ & 3 & 0,163 & 2 & 0,37 & 0,32 \\
\hline Myrrhinium atropurpureum Schott & JCB 122 & BPU & 5 & 0,034 & 5 & 0,17 & 0,30 \\
\hline Psidium cattleyanum $\mathrm{Sab}$. & $\mathrm{NC}$ & EAD & 5 & 0,041 & 4 & 0,19 & 0,27 \\
\hline Myrciaria tenella (DC.) Berg. & JCB 270 & EAD & 1 & 0,005 & 1 & 0,03 & 0,05 \\
\hline RUBIACEAE & & & 73 & 0,769 & 51 & 3,08 & 3,90 \\
\hline Guettarda uruguensis Cham. \& Schltdl. & JCB 405 & BPU & 48 & 0,535 & 30 & 2,08 & 2,48 \\
\hline Chomelia obtusa Cham. \& Schltdl. & JCB 283 & EAD & 20 & 0,180 & 17 & 0,79 & 1,14 \\
\hline Randia armata (Sw.) DC. & JCB 245 & EAD & 5 & 0,054 & 4 & 0,21 & 0,28 \\
\hline RUTACEAE & & & 42 & 1,512 & 23 & 3,75 & 3,66 \\
\hline Helietta apiculata Benth. & JCB 314 & BPU & 41 & 1,504 & 31 & 3,71 & 3,60 \\
\hline Zanthoxylum fagara (L.) Sarg. & JCB 264 & $\mathrm{BPU}$ & 1 & 0,008 & 1 & 0,04 & 0,06 \\
\hline MYRSINACEAE & & & 68 & 0,593 & 39 & 2,64 & 3,21 \\
\hline Myrsine loefgrenii (Mez) Otegui & JCB 234 & BPU & 58 & 0,368 & 34 & 2,00 & 2,57 \\
\hline Myrsine parvula (Mez) Otegui & $\mathrm{NC}$ & BPU & 10 & 0,225 & 6 & 0,64 & 0,64 \\
\hline BORAGINACEAE & & & 46 & 1,240 & 27 & 3,34 & 3,21 \\
\hline Patagonula americana $\mathrm{L}$. & JCB 236 & BPU & 46 & 1,240 & 27 & 3,34 & 3,21 \\
\hline FABACEAE & & & 47 & 0,709 & 33 & 2,36 & 3,07 \\
\hline Machaerium paraguariense Hassl. & JCB 416 & BPU & 15 & 0,376 & 15 & 1,03 & 1,23 \\
\hline Dalbergia frutescens (Vell.) Britton & JCB 306 & BPU & 23 & 0,165 & 17 & 0,83 & 1,17 \\
\hline Albizia niopoides (Spruce ex Benth.) Burkart & JCB 330 & BPU & 4 & 0,116 & 4 & 0,30 & 0,35 \\
\hline Parapiptadenia rigida (Benth.) Brenan & $\mathrm{NC}$ & BPU & 3 & 0,035 & 3 & 0,13 & 0,20 \\
\hline Inga vera Willd & $\mathrm{NC}$ & BPU & 1 & 0,015 & 1 & 0,05 & 0,07 \\
\hline Acacia bonariensis Gill. & NC & BPU & 1 & 0,002 & 1 & 0,02 & 0,05 \\
\hline VERBENACEAE & & & 38 & 1,259 & 25 & 3,19 & 3,03 \\
\hline Vitex megapotamica (Spreng.) Mold. & JCB 309 & EAD & 33 & 0,997 & 20 & 2,59 & 2,45 \\
\hline Cytharexylum montevidense (Spreng.) Mold. & JCB 344 & BPU & 5 & 0,262 & 5 & 0,60 & 0,58 \\
\hline SAPOTACEAE & & & 32 & 0,738 & 23 & 2,09 & 2,23 \\
\hline Chrysophyllum marginatum (Hook. \& Arn.) Radlk. & JCB 381 & $\mathrm{BPU}$ & 32 & 0,738 & 23 & 2,09 & 2,23 \\
\hline POLYGONACEAE & & & 22 & 0,676 & 17 & 1,75 & 1,78 \\
\hline Ruprechtia laxiflora Meiss. & JCB 208 & BPU & 22 & 0,676 & 17 & 1,75 & 1,78 \\
\hline LAURACEAE & & & 25 & 0,389 & 22 & 1,29 & 1,66 \\
\hline Ocotea pulchella Mart. & JCB 312 & EAD & 25 & 0,389 & 22 & 1,29 & 1,66 \\
\hline FLACOURTIACEAE & & & 27 & 0,378 & 20 & 1,31 & 1,60 \\
\hline Casearia decandra Jacq. & JCB 277 & BPU & 21 & 0,319 & 15 & 1,07 & 1,25 \\
\hline Casearia sylvestris $\mathrm{Sw}$. & $\mathrm{NC}$ & EAD & 4 & 0,023 & 3 & 0,13 & 0,20 \\
\hline
\end{tabular}


Tabela 1 (continuação)

\begin{tabular}{|c|c|c|c|c|c|c|c|}
\hline Família/Espécie & $\mathrm{NC}$ & CG & DA & DoA & FA & $\mathrm{VC}$ & VI \\
\hline \multicolumn{8}{|l|}{ FLACOURTIACEAE } \\
\hline Banara tomentosa $\mathrm{Clos}$ & JCB 338 & BPU & 2 & 0,036 & 2 & 0,11 & 0,15 \\
\hline SAPINDACEAE & & & 25 & 0,316 & 19 & 1,15 & 1,52 \\
\hline Allophylus edulis (A. St.-Hil.) Radlk. ex Warm. & JCB 214 & EAD & 20 & 0,261 & 16 & 0,94 & 1,20 \\
\hline Matayba eleagnoides Radlk. & JCB 204 & EAD & 5 & 0,055 & 5 & 0,21 & 0,32 \\
\hline ANNONACEAE & & & 16 & 0,202 & 10 & 0,74 & 0,85 \\
\hline Rollinia salicifolia Schltdl. & JCB 324 & BPU & 16 & 0,202 & 10 & 0,74 & 0,85 \\
\hline LOGANIACEAE & & & 12 & 0,176 & 11 & 0,60 & 0,80 \\
\hline Strychnos brasiliensis (Spreng.) Mart. & JCB 219 & EAD & 12 & 0,176 & 11 & 0,60 & 0,80 \\
\hline MALVACEAE & & & 7 & 0,239 & 7 & 0,60 & 0,65 \\
\hline Luehea divaricata Mart. \& Zucc. & $\mathrm{NC}$ & EAD & 7 & 0,239 & 7 & 0,60 & 0,65 \\
\hline ASTERACEAE & & & 5 & 0,099 & 5 & 0,30 & 0,38 \\
\hline Gochnatia polymorpha Cabr. & JCB 375 & EAD & 5 & 0,099 & 5 & 0,30 & 0,38 \\
\hline SYMPLOCACEAE & & & 4 & 0,143 & 4 & 0,35 & 0,38 \\
\hline Symplocos uniflora (Pohl.) Benth. & JCB 285 & EAD & 4 & 0,143 & 4 & 0,35 & 0,38 \\
\hline Intederminada & & & 4 & 0,124 & 3 & 0,32 & 0,32 \\
\hline CELTIDACEAE & & & 5 & 0,022 & 5 & 0,15 & 0,28 \\
\hline Celtis iguanae (Jacq.) Spreng. & JCB 242 & BPU & 5 & 0,022 & 5 & 0,15 & 0,28 \\
\hline EBENACEAE & & & 3 & 0,092 & 3 & 0,24 & 0,27 \\
\hline Diospyros inconstans Jacq. & JCB 355 & BPU & 3 & 0,092 & 3 & 0,24 & 0,27 \\
\hline SALICACEAE & & & 1 & 0,068 & 1 & 0,15 & 0,13 \\
\hline Salix humboldtiana Willd. & $\mathrm{NC}$ & EAD & 1 & 0,068 & 1 & 0,15 & 0,13 \\
\hline ANACARDIACEAE & & & 1 & 0,016 & 1 & 0,05 & 0,07 \\
\hline Schinus terebinthifolius Raddi & $\mathrm{NC}$ & EAD & 1 & 0,016 & 1 & 0,05 & 0,07 \\
\hline SOLANACEAE & & & 1 & 0,014 & 1 & 0,05 & 0,07 \\
\hline Solanum sanctae-catharinae Dunal & $\mathrm{NC}$ & EAD & 1 & 0,014 & 1 & 0,05 & 0,07 \\
\hline MELIACEAE & & & 1 & 0,006 & 1 & 0,03 & 0,06 \\
\hline Trichillia catigua A. Juss. & JCB 224 & BPU & 1 & 0,006 & 1 & 0,03 & 0,06 \\
\hline ERYTHROXYLACEAE & & & 1 & 0,002 & 1 & 0,02 & 0,05 \\
\hline Erythroxylum deciduum A. St.-Hil. & JCB 351 & EAD & 1 & 0,002 & 1 & 0,02 & 0,05 \\
\hline
\end{tabular}

perfizeram $50,37 \%$ do valor total. $\mathrm{O}$ valor de cobertura (VC) seguiu a mesma ordem de VI, para as primeiras espécies.

Observando-se o Valor de Importância de cada família, destaca-se a família Euphorbiaceae, que embora possua apenas três espécies, apresentou o

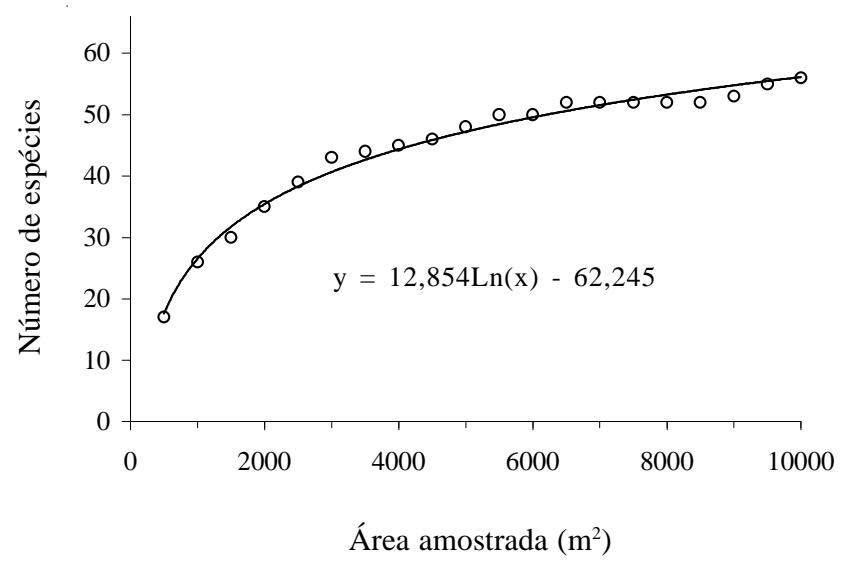

Figura 1. Curva espécies/área de um trecho de floresta ribeirinha do arroio Passo das Tropas, Santa Maria, RS, Brasil. maior VI (Tab. 1). Por outro lado, Myrtaceae, que apresentou a maior riqueza em espécies, aparece em segundo lugar, considerando-se este parâmetro. Em terceira posição está Rubiaceae, seguida por Rutaceae, com o quarto maior VI e apenas duas espécies. Fabaceae teve poucos indivíduos amostrados, ficando na sétima posição em VI, embora possuísse o segundo maior número de espécies (6).

Cerca de metade dos indivíduos (52\%) estavam na menor classe de diâmetro (Fig. 2), que é composta pelos indivíduos de espécies características do subbosque e aqueles regenerantes das espécies do dossel superior. A segunda classe, entre 10 e $15 \mathrm{~cm}$, abriga $21,77 \%$ dos indivíduos amostrados. A partir da terceira classe, ocorre redução gradativa no número de indivíduos, o que já era esperado, pois há uma relação inversa entre número e tamanho (porte) dos indivíduos amostrados, conseqüência da dinâmica florestal (Oliveira et al. 2001).

A distribuição de freqüência das classes de altura é apresentada na Fig. 3. Pode-se observar a 


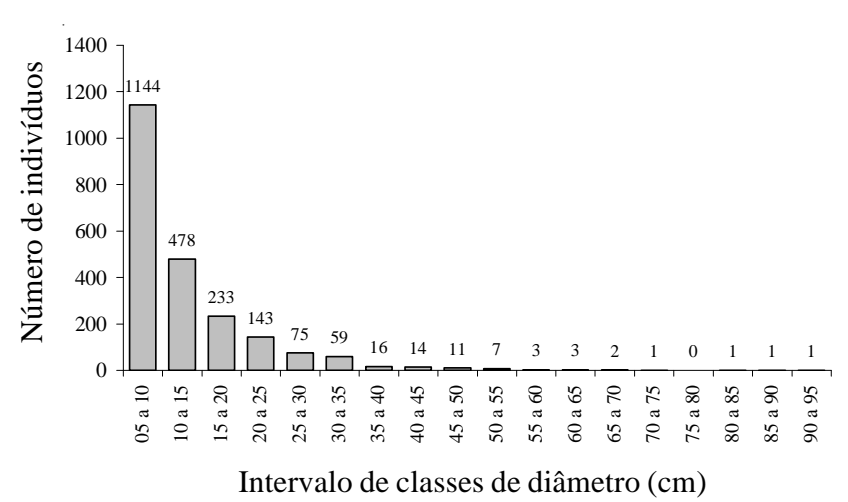

Figura 2. Distribuição dos indivíduos amostrados na floresta ribeirinha do arroio Passo das Tropas, Santa Maria, RS em intervalos de classes de diâmetro.

inexistência de extratos distintos e poucas árvores emergentes. No entanto, algumas espécies possuem características de arvoretas, com indivíduos de baixas alturas como Gymnanthes concolor, Chomelia obtusa, Sebastiania brasiliensis e Myrsine loefgrenii, que representaram $40,7 \%$ dos indivíduos amostrados. De maneira geral, a mata ribeirinha estudada pode ser considerada como de baixa altura, com poucos indivíduos ultrapassando $15 \mathrm{~m}$ conforme observado na Fig. 4. Dentre as espécies que marcam a fisionomia da floresta, estão Vitex megapotamica, Matayba eleagnoides, Albizia niopoides, Helietta apiculata, Ocotea pulchella, Luehea divaricata e Ruprechtia laxiflora.

Foram encontradas 137 árvores mortas, correspondendo a 5,87\% dos indivíduos amostrados, sendo que o número de árvores mortas variou de zero a seis por parcela. Este valor é o maior já encontrado em levantamentos no Estado, mas não difere significativamente dos resultados já observados. Jarenkow (1994) ao comparar duas áreas de mata de encosta encontrou valores de $5,1 \%$ e $1,9 \%$; Vasconcellos et al. (1992) trabalhando no Parque Estadual do Turvo, encontraram 4,6\% de árvores mortas e R.V. Kilca (dados não publicados), numa floresta ribeirinha da Planície Costeira do Estado, encontrou $4,15 \%$ de árvores mortas.

A presença de árvores mortas é característica natural da floresta e afeta, pela formação de clareiras, o recrutamento de novos indivíduos de diferentes espécies (Oliveira et al. 2001; Denslow 1987; Whitmore 1989; Tabarelli et al. 1999) e, conseqüentemente, a distribuição espacial dos indivíduos (Dale 1997). Algumas espécies amostradas, como Rollinia salicifolia, Machaerium paraguariense e Citharexylum montevidense apresentaram somente

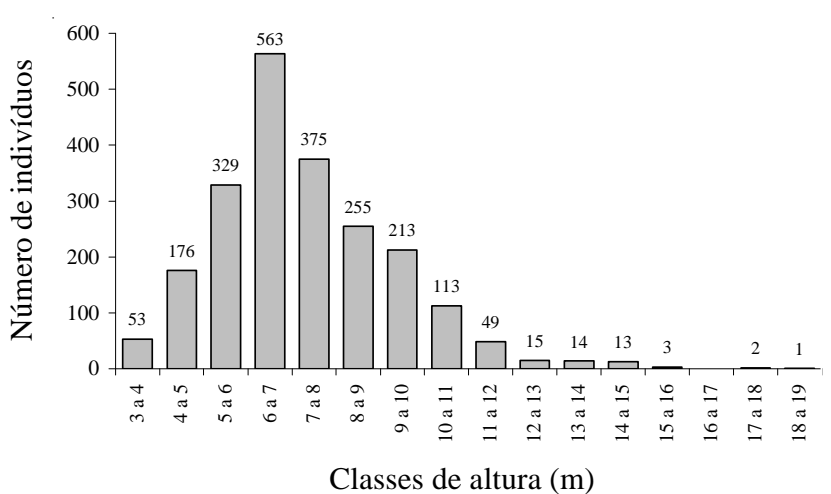

Figura 3. Distribuição de freqüência de classes de altura dos indivíduos amostrados na floresta ribeirinha do arroio Passos das Tropas, Santa Maria, RS.

indivíduos de grande porte, o que pode indicar problemas de regeneração ou justamente a dependência da formação de clareiras para ocorrer recrutamento.

$\mathrm{O}$ índice de diversidade de Shannon (H') foi de 2,73 (nats/ind.), sendo considerado intermediário quando comparado a valores encontrados em outras áreas da região (Jarenkow \& Waechter 2001; Longhi et al. 1999; Daniel 1991; Nascimento et al. 1998; Longhi et al. 2000). No entanto, é um valor muito baixo se comparado aos encontrados para matas de galeria da região Central e Sudeste do Brasil (van der Berg \& Oliveira Filho 2000; Botrel et al. 2002; Bertani et al. 2001; Martins 1991; Ivanauskas et al. 1997). Dois fatores associados podem estar causando a diminuição no índice de diversidade, comparando-se a levantamentos realizados naquelas regiões: redução no número de espécies no sentido norte-sul, relacionado a fatores biogeográficos e, diminuição na equabilidade (J') de Pielou, o que indicaria mudanças nos processos ecológicos atuais. A equabilidade de Pielou para o presente estudo ficou em 0,69. Este valor indica que algumas espécies estão com altas densidades, sendo que outras possuem baixos números de indivíduos. No entanto, estas hipóteses são ainda preliminares, pois a detecção de padrões de diversidade requer maiores estudos nas formações ribeirinhas do Rio Grande do Sul.

Nove espécies $(15,78 \%)$ apresentaram apenas um indivíduo amostrado. Conforme Martins (1991), espécies com baixos valores de densidade e freqüência são consideradas raras. Entretanto, esta consideração deve ser feita apenas para a área de estudo, sem generalizações, pois estas espécies podem ocorrer em elevada densidade, mesmo em áreas próximas, como Salix humboldtiana, Schinus terebinthifolius, Inga vera, Trichillia catigua, Myrciaria tenella e Erythroxylum deciduum. Em contrapartida, outras 


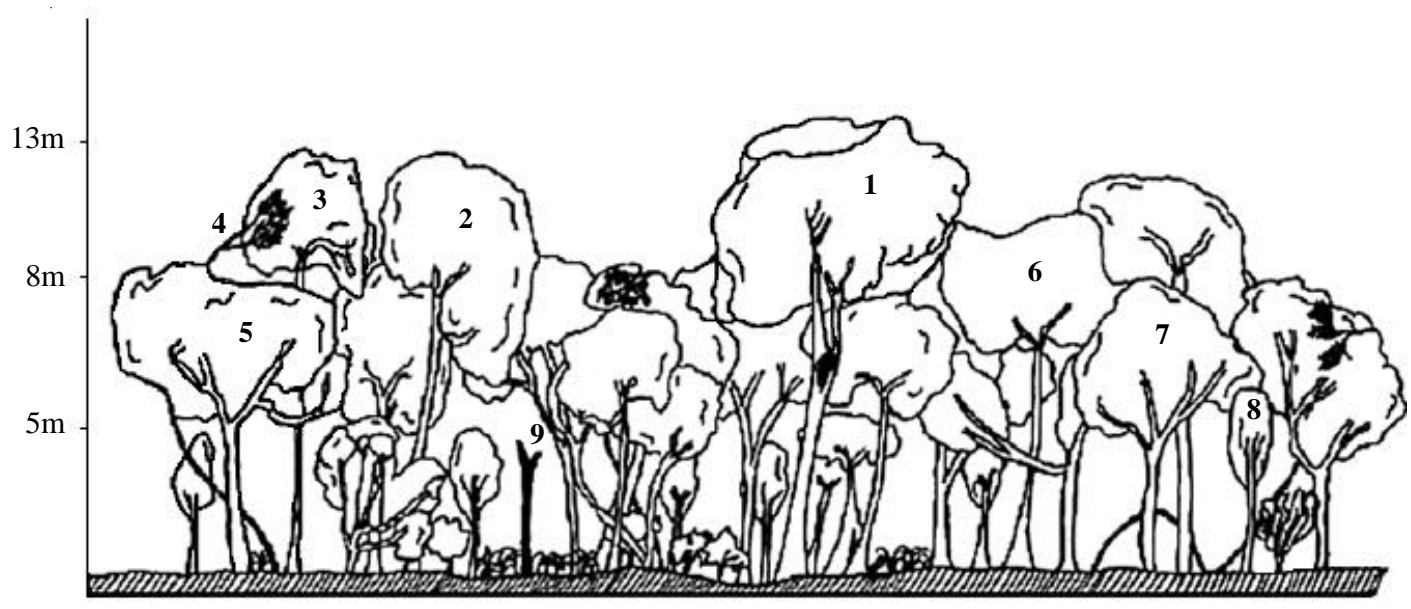

Figura 4. Perfil esquemático da vegetação, confeccionado nas parcelas n. 39-40, instalada na floresta ribeirinha do arroio Passo das Tropas, Santa Maria, RS, Brasil. 1. Helietta apiculata. 2. Ocotea pulchella. 3. Sebastiania commersoniana. 4. Dasyphyllum spinescens (liana). 5. Plinia rivularis. 6. Patagonula americana. 7. Myrsine loefgrenii. 8. Gymnanthes concolor. 9. Morta.

espécies, com elevada freqüência em levantamentos na região, não foram encontradas no presente estudo, o que poderia ser resultado de cortes seletivos pretéritos. Desta forma, ao considerar-se que uma espécie é rara, dever-se-ia verificar se isto é caráter inerente à biologia da espécie ou se é um evento da comunidade em estudo (estádio de desenvolvimento da sinúsia, formação de clareiras). Em se tratando de florestas ribeirinhas, características intrínsecas do meio, como remoção de serapilheira e encharcamento prolongado do solo poderiam determinar maior ou menor ocorrência de uma determinada espécie (Rodrigues \& Shepherd 2000).

A observação do contingente migratório revelou que $57 \%$ das espécies pertencem ao contingente oeste, ou seja, são provenientes da bacia Paraná-Uruguai, enquanto que $41 \%$ das espécies são de ampla distribuição, e apenas uma espécie, Eugenia hyemalis provém do contingente leste. Por outro lado, ao analisar-se o número de indivíduos em cada categoria, o quadro é modificado. Espécies de ampla distribuição contribuem com 1.372 indivíduos, ou seja, $62,5 \%$ dos indivíduos amostrados. Já as espécies provenientes da Bacia Paraná-Uruguai contribuem com 781 indivíduos (35,5\%). Foram amostrados 42 indivíduos de Eugenia hyemalis, ou seja, $2 \%$ dos indivíduos. Jarenkow \& Waechter (2001), em uma floresta de encosta no centro do Estado, encontraram valores semelhantes aos do presente estudo, onde o elemento de ampla distribuição possuiu o maior número de indivíduos amostrados e menor número de espécies. Já as espécies do contingente oeste, por outro lado, contribuíram com a maior riqueza e menor número de indivíduos amostrados. Quanto à rota do corredor atlântico, os autores encontraram reduzido número de espécies e número intermediário de indivíduos. Já no presente estudo, a proporção de espécies e indivíduos provindos do corredor atlântico é muito reduzida, não contribuindo significativamente na composição da floresta.

Por meio da análise florístico-estrutural e fitogeográfica realizada na floresta ribeirinha em questão, pode-se afirmar que a mesma possui diversidade florística intermediária, comparando-se com outros levantamentos. Isto provavelmente seria resultado dos diferentes fatores que interferem sobre o mosaico ribeirinho, influenciado pela predominância dos elementos florísticos provindos do contingente oeste e por espécies de ampla distribuição.

\section{Agradecimentos}

Os autores agradecem ao Fundo de Incentivo à Pesquisa - FIPE/UFSM e ao Programa Especial de Treinamento - PET/BIOLOGIA, pelo apoio financeiro; aos revisores anônimos, pelas correções e recomendações.

\section{Referências bibliográficas}

APG (ANGIOSPEM PHYLOGENY GROUP). 1998. An ordinal classification for the families of flowering plants. Annals of the Missouri Botanical Garden 85: 531-553.

Berg, E. van der \& Oliveira-Filho, A.T. 2000. Composição florística e estrutura fitossociológica de uma floresta ripária em Itutinga, MG, e comparação com outras áreas. Revista Brasileira de Botânica 23(3): 231-253. 
Bertani, D.F.; Rodrigues, R.R.; Batista, J.L.F. \& Shepherd, G.J. 2001. Análise temporal da heterogeneidade florística e estrutural em uma floresta ribeirinha. Revista Brasileira de Botânica 24(1): 11-23.

Botrel, R.; Oliveira Filho, A.T.; Rodrigues, L.A. \& Curi, N. 2002. Influência do solo e topografia sobre as variações da composição florística e estrutura da comunidade arbóreo-arbustiva de uma floresta estacional semidecidual em Ingaí, MG. Revista Brasileira Botânica 25(2): 195-213.

Brower, J.E. \& Zar, J.H. 1977. Field and laboratory methods for general ecology. W.C. Brown Company Publishers, Iowa

Dale, M.R.T. 1997. Spatial pattern analysis in plant ecology. Cambridge Studies in Ecology, Cambridge.

Daniel, A. 1991. Estudo fitossociológico arbóreo/arbustivo da mata ripária da bacia hidrográfica do Rio dos Sinos, RS. Pesquisas, Série Botânica 41: 5-199.

Denslow, J.S. 1987. Tropical rainforest gaps and tree species diversity. Annual Review of Ecology and Systematics 18: $432-451$

Ivanauskas, N.M.; Rodrigues, R.R. \& Nave, A.G. 1997. Aspectos ecológicos de um trecho de floresta de brejo em Itatinga, SP. Revista Brasileira de Botânica 20(2): 139-153.

Jarenkow, J.A. 1994. Estudo fitossociológico comparativo entre duas áreas com mata de encosta no Rio Grande do Sul. Tese doutorado, Universidade Federal de São Carlos, São Carlos.

Jarenkow, J.A. Baptista \& L.R.M. 1987. Composição florística e estrutura da mata com araucária na Estação Ecológica de Aracuri, Esmeralda, Rio Grande do Sul. Napaea 3: 9-18.

Jarenkow, J.A. \& Waechter, J.L. 2001. Composição, estrutura e relações florísticas do componente arbóreo de uma floresta estacional no Rio Grande do Sul, Brasil. Revista Brasileira de Botânica 24(3): 263-272.

Klein, R.M. 1972. Árvores nativas da Floresta Subtropical do Alto Uruguai. Sellowia 24: 9-62.

Leite, E.J. 2001. Spatial distribution patterns of riverine taxa in Brasília, Brazil. Forest Ecology and Management 140: 257-264.

Lemos, R.C.; Azolin, M.D. \& Abrao, P.R. \& Santos, M.C.L. 1973. Levantamento de reconhecimento dos solos do Estado do Rio Grande do Sul. Ministério da Agricultura - Departamento Nacional de Pesquisas Agropecuárias Divisão de Pesquisa Pedológica, Recife.

Longhi, S.J.; Nascimento, A.R.T.; Fleig, F.D.; Della-Flora, J.B.; Freitas, R.A. \& Charão, L.W. 1999. Composição florística e estrutura da comunidade arbórea de um fragmento florestal no município de Santa Maria-Brasil. Ciência Florestal 9(1): 115-133.

Longhi, S.J.; Araujo, M.M.; Kelling, M.B.; Hoppe, J.M.; Müller, I. \& Borsoi, G.A. 2000. Aspectos fitossociológicos de fragmento de floresta estacional decidual, Santa Maria, RS. Ciência Florestal 10(2): 59-74.

Maluf, J.R.T. 2000. Nova classificação climática do Estado do Rio Grande do Sul. Revista Brasileira de Agrometeorologia 8(1): 141-150.
Martins, F.R. 1991. Estrutura de uma floresta mesófila. Editora da UNICAMP, Campinas.

Moreno, J.A. 1961. Clima do Rio Grande do Sul. Secretaria da Agricultura - Div. Terras e Colonização, Porto Alegre.

Moreno, M.I.C. \& Schiavini, I. 2001. Relação entre vegetação e solo em um gradiente florestal na Estação Ecológica do Panga, Uberlândia (MG). Revista Brasileira de Botânica 24(4): 537-544.

Nascimento, A.R.T.; Longhi, S.J.; Alvarez Filho, A. \& Gomes, G.S. 2000. Análise da diversidade florística e dos sistemas de dispersão de sementes em um fragmento florestal da região central do Rio Grande do Sul, Brasil. Napaea 12: 49-67.

Oliveira, R.J.; Mantovani, W. \& Melo, M.M.R.F. 2001. Estrutura do componente arbustivo-arbóreo da floresta atlântica de encosta, Peruíbe, SP. Acta Botanica Brasilica 15(3): 391-412.

Oliveira Filho, A.T.; Ratter, J.A. \& Shepherd, G.J. 1990. Floristic composition and community structure of a central Brazilian gallery forest. Flora 184: 103-117.

Rambo, B. 1949. A flora de Cambará. Anais Botânicos do Herbário Barbosa Rodrigues 1(1): 111-135.

Rambo, B. 1951. A imigração da selva higrófila no Rio Grande do Sul. Anais Botânicos do Herbário Barbosa Rodrigues 3: $55-91$

Rambo, B. 1961. Migration routes of the south brazilian rain forest. Pesquisas, Série Botânica 12: 1-54.

Reitz, R.; Klein, R.M. \& Reis, A. 1983. Projeto madeira do Rio Grande do Sul. Sellowia 34/35: 1-525.

Ribeiro, J.F. \& Walter, B.M.T. 1998. Fitofisionomias do Bioma Cerrado. Pp. 89-16. In: S.M. Sano \& S.P. Almeida (eds.). Cerrado: Ambiente e flora. EMBRAPA CPAC, Planaltina.

Rodrigues, R.R. 2000. Uma discussão nomenclatural das formações ciliares. Pp. 91-100. In: R.R. Rodrigues \& H.F. Leitão-Filho (eds.). Matas ciliares: conservação e recuperação. EDUSP/FAPESP, São Paulo.

Rodrigues, R.R. \& Nave, A.G. 2000. Heterogeneidade florística das matas ciliares. Pp. 45-71. In: R.R. Rodrigues \& H.F. Leitão-Filho (eds.). Matas ciliares: conservação e recuperação. EDUSP/FAPESP, São Paulo.

Rodrigues, R.R. \& Shepherd, G.J. 2000. Fatores condicionantes da vegetação ciliar. Pp. 101-107. In: E.E. Rodrigues \& H.F. Leitão-Filho (eds.). Matas ciliares: conservação e recuperação. EDUSP/FAPESP, São Paulo.

Shepherd, G.J. 1995. FITOPAC 1. Manual de usuário. Departamento de Botânica, UNICAMP.

Tabarelli, M. 1992. Flora arbórea da floresta estacional baixomontana no município de Santa Maria, RS, Brasil. Pp. 260-268. In: Anais do II Congresso Nacional sobre Essências Nativas. Instituto Florestal, Piracicaba.

Tabarelli, M.; Mantowani, W. \& Peres, C.A. 1999. Effects of habitat fragmentation on plant guild structure in the montane Atlantic Forest of southeastern Brazil. Biological Conservation 91: 119-127.

Toniato, M.T.Z.; Rodríguez, R.R. \& Leitão Filho, H.F. 1998. Fitossociologia de um remanescente de floresta higrófila (mata de brejo) em Campinas, São Paulo. Revista Brasileira de Botânica 21(2): 197-210. 
Vasconcellos, J.M.O.; Dias, L.L.; Silva, C.P. \& Sobral, M 1992. Fitossociologia de uma área de mata subtropical no Parque Estadual do Turvo, RS. Revista do Instituto Florestal 4: 252-259.
Veloso, H.P.; Rangel Filho, A.L.R. \& Lima, J.C.A. 1991. Classificação da vegetação brasileira adaptada a um sistema universal. IBGE, Rio de Janeiro.

Whitmore, T.C. 1989. Canopy gaps and the two major groups of forest trees. Ecology 70(3): 536-538. 